\title{
ChemComm
}

Check for updates

Cite this: Chem. Commun., 2019 55,3594

Received 1st December 2018 Accepted 22nd February 2019

DOI: $10.1039 / \mathrm{c} 8 \mathrm{cc} 09571 \mathrm{j}$

rsc.li/chemcomm

\section{Controlling the crystalline structure of imine-linked 3D covalent organic frameworks $\dagger$}

\author{
Danyon M. Fischbach, Grace Rhoades, Charlie Espy, Fallon Goldberg and \\ Brian J. Smith (D)*
}

\begin{abstract}
We explore the growth mechanism of 3D Covalent Organic Frameworks (COFs) using imine-linked model systems. We confirm that 3D imine COFs crystalize through an amorphous polymer intermediate, whose conditional state is key for preferential generation of the ideal porous structure over the collapsed hydrated form.
\end{abstract}

Covalent organic frameworks (COFs) are crystalline, porous polymer networks with programmable pore sizes and high internal surface areas. ${ }^{1-7}$ The readily designable structure of COFs, based on monomer choice, is ideal for a range of applications ${ }^{8}$ including separations, ${ }^{9,10}$ catalysis, ${ }^{11-13}$ energy devices, ${ }^{14-18}$ and gas storage. ${ }^{19,20}$ COF synthesis relies on self-assembly via reversible covalent linkages, which allow for the correction of structural defects during formation to generate the crystalline framework. Of the variety of linkages employed, imine COFs are particularly attractive due to their increased water stability over first generation boronate ester COFs. ${ }^{21}$ The two main structural categories of COFs are two- and three-dimensional systems: 2D COFs consist of discrete planar sheets with non-covalent interlayer stacking, whereas 3D COFs are interconnected, fully covalent systems. This threedimensional connectivity provides more control over the overall network, as well as a broader range of potential structures. Despite the fact that 3D COFs have multiple reported single crystals ${ }^{22,23}$ and have higher potential surface areas than their 2D counterparts, $2 \mathrm{D}$ frameworks have thus far achieved broader application. This is due in part to a better mechanistic understanding of the 2D COF formation processes. ${ }^{24-26}$ An increased understanding of 3D COF growth mechanisms will provide greater control over functionalization and design of next-generation materials.

We chose COF-300 as a model system for studying 3D iminelinked COF formation (Fig. 1). ${ }^{27}$ Despite the adamantane-like structure predictable from the rigid monomers tetrakis(4aminophenyl)methane (TAPM) and terephthaldehyde (PDA),

Department of Chemistry, Bucknell University, Rooke Science Center, Lewisburg, Pennsylvania 17837, USA. E-mail: brian.smith@bucknell.edu

$\dagger$ Electronic supplementary information (ESI) available: Experimental methods and additional characterization. See DOI: 10.1039/c8cc09571j
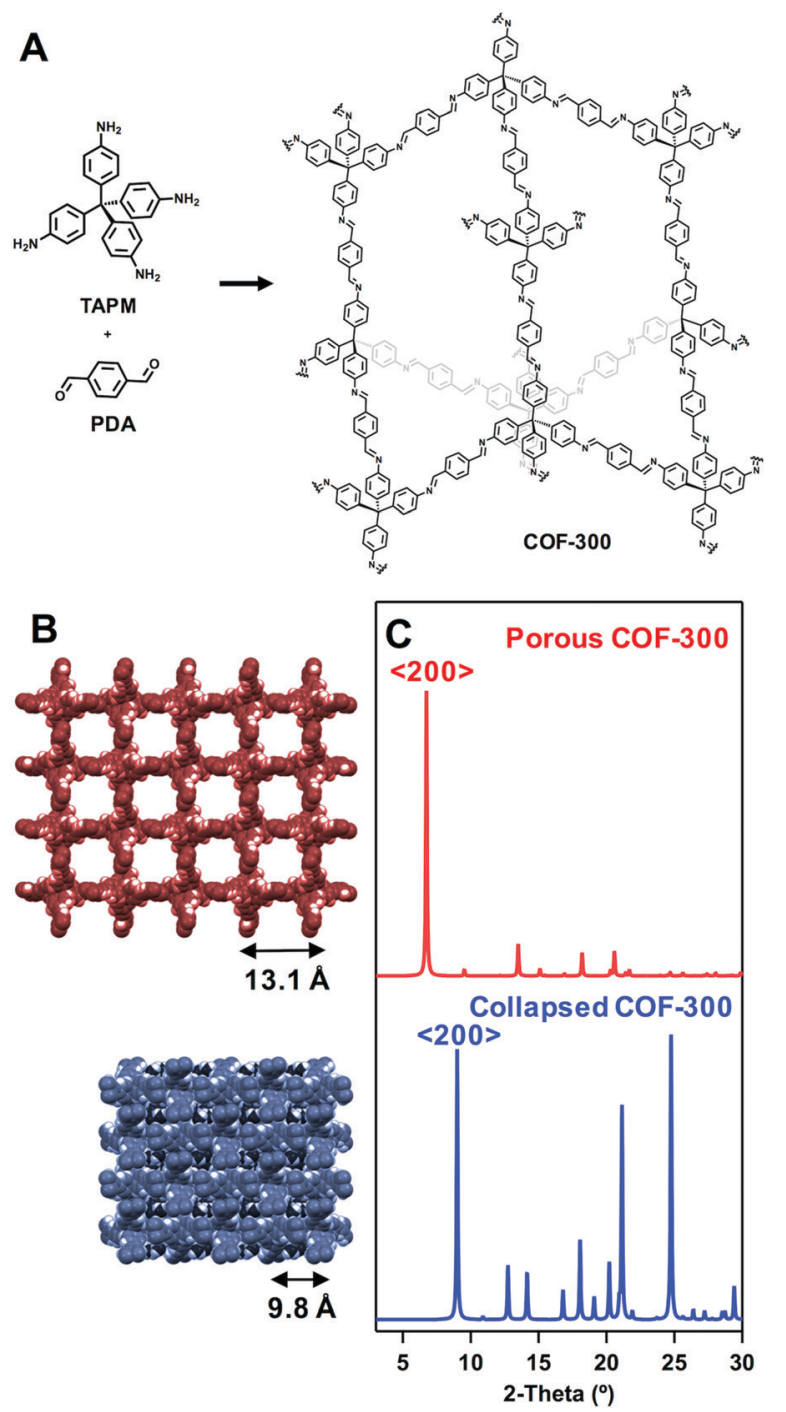

Fig. 1 (A) Scheme of imine-linked COF-300 connectivity, (B) space filling models of the crystal structures of interpenetrated porous and collapsed COF-300, initially reported in ref. 22 , highlighting the $\langle 200\rangle$ spacing, and (C) PXRD patterns generated from the reported crystal structures. 
certain synthetic challenges remain, including network interpenetration and structural distortion. It was recently shown that COF-300 can be crystalized as either the expected seven-fold interpenetrated, porous network or as a collapsed, hydrated form. ${ }^{22}$ Even with the high degree of interpenetration, COF-300 retains $1 \mathrm{D}$ pores corresponding to the spacing of the $\langle 200\rangle$ Miller planes. The reported hydrated form shows a collapse of the $\langle 200\rangle$ spacing from $13.1 \AA$ to $9.8 \AA$, as well as crystallographically observed water molecules filling the pores. While both structures are known, the key conditions controlling selective synthesis have not yet been established.

The growth of COF-300 was first evaluated from fully homogeneous starting conditions. Heating the monomer solution (1.8:1 dioxane: $3 \mathrm{M}$ acetic acid) at $90{ }^{\circ} \mathrm{C}$ for two days at atmospheric pressure results in high yields of crystalline COF300 in the collapsed form $(85 \%)$. The structure was confirmed by PXRD, with the $\langle 200\rangle$ diffraction peak at $8.95^{\circ} 2 \theta$, and shows no evidence of the expected porous form (Fig. 2A). $\mathrm{N}_{2}$ sorption studies reveal that collapsed COF-300 has an extremely low surface area, $c a .20 \mathrm{~m}^{2} \mathrm{~g}^{-1}$, which is dramatically below values expected for porous COF-300. All activation attempts indicate that it is extremely difficult to convert the collapsed system into porous COF-300 via standard COF preparative conditions (Fig. S1 and S2, ESI $\dagger$ ). Thermogravimetric analysis of the collapsed form at atmospheric pressure shows no appreciable change in mass between $150{ }^{\circ} \mathrm{C}$ and degradation at $500{ }^{\circ} \mathrm{C}$ (Fig. S6, ESI $\dagger$ ). Correspondingly, high vacuum evacuation at elevated temperatures shows no conversion to the porous COF-300 structure. Attempts to synthesize COF-300 in the absence of added water still result in formation of the collapsed form, suggesting that the water released by initial imine formation is sufficient to induce structural distortion (Fig. S5, ESI $\dagger$ ). The low surface area of the collapsed form is consistent with the strong binding of water within the distorted crystalline structure and limits application that rely on the internal porosity. As collapsed COF-300 cannot be easily activated into the porous COF-300 form, it is critically important to develop conditions that reliably yield the target porous structure.

A mechanistic analysis of the early stages of growth from homogeneous conditions was performed to identify intermediate structures. It has been established for $2 \mathrm{D}$ imine $\mathrm{COFs},{ }^{26}$ and proposed for $3 \mathrm{D}$ imine COFs, ${ }^{22}$ that the growth proceeds through an intermediate amorphous gel state which is capable of directly rearranging into the crystalline form over time. For COF-300, upon initial addition of PDA to the TAPM monomer solution, solids rapidly form at room temperature in high yields $(>70 \%)$. The isolated material is amorphous (Fig. 2C) and has a low surface area of $c a .50 \mathrm{~m}^{2} \mathrm{~g}^{-1}$ (Fig. S8, ESI $\dagger$ ). IR spectroscopy of the resulting amorphous solid is distinct from the crystalline COF-300, with the peak at $1698 \mathrm{~cm}^{-1}$ suggesting residual unreacted functional groups (Fig. S12, ESI $\dagger$ ). To establish that the amorphous material is capable of transforming into the crystalline species, the isolated solid was purified and re-exposed to COF-300 growth conditions, in the absence of any additional monomer. Heating the suspension under standard homogenous growth conditions ( $90{ }^{\circ} \mathrm{C}, 2$ days) yields crystalline COF-300. Remarkably, amorphous regrowth yields the ideal porous COF-300 form, confirmed by PXRD with the major $\langle 200\rangle$ diffraction peak at $6.65^{\circ} 2 \theta$ (Fig. 2D). The regrowth COF-300 possesses a high surface area $\left(c a .1000 \mathrm{~m}^{2} \mathrm{~g}^{-1}\right)$, approximately 50-fold higher than the collapsed form. Also in contrast to the strong water binding of the collapsed form, porous COF-300 treated with either 1,4-dioxane or water is
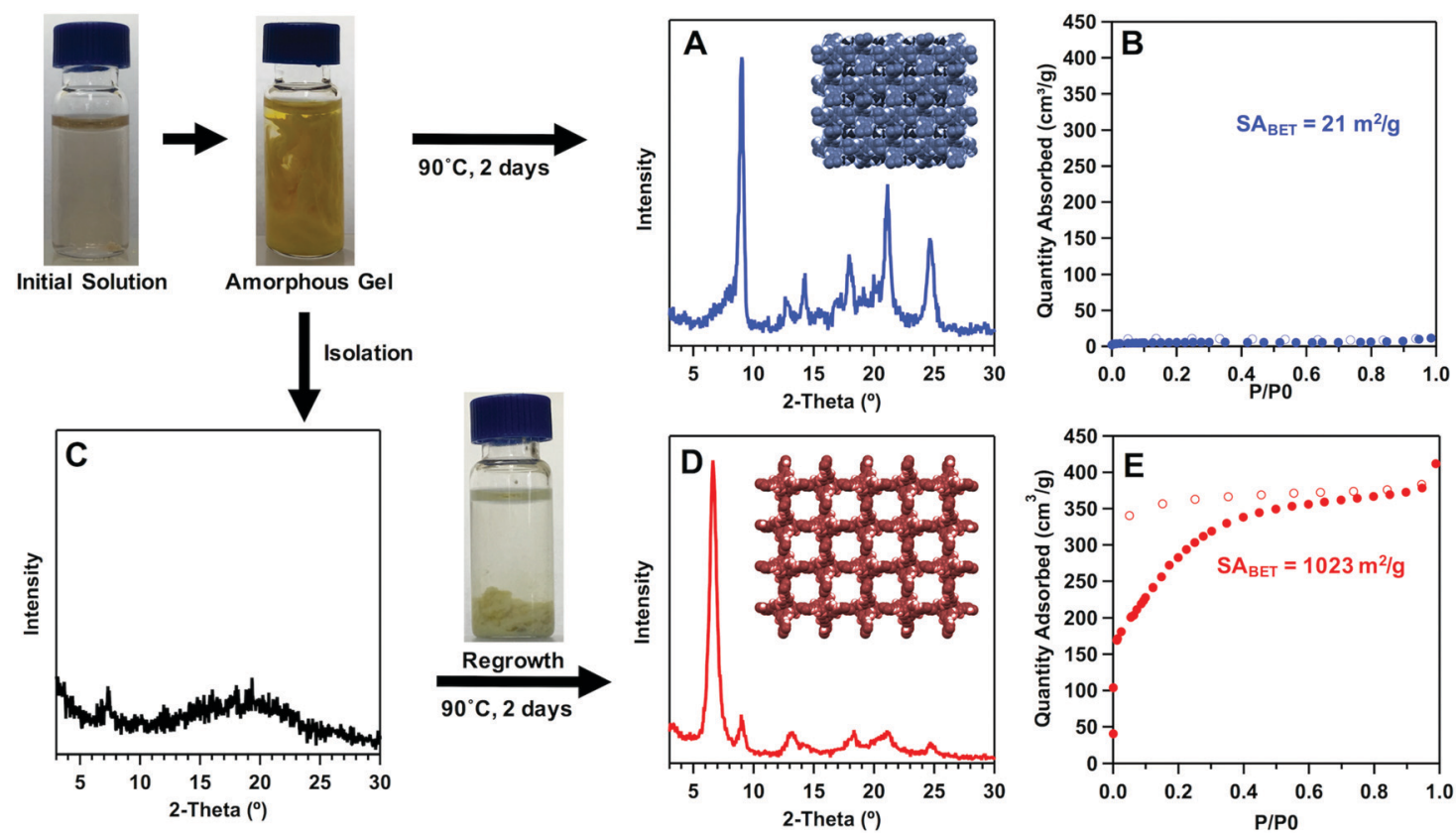

Fig. 2 Synthesis of COF-300 through both direct homogeneous conditions and isolation and regrowth of initial amorphous gel. Photos of suspensions prior to heating. Direct synthesis yields collapsed COF-300 based on (A) PXRD and (B) $N_{2}$ sorption. (C) The amorphous intermediate re-exposed to growth conditions yields porous COF-300 based on (D) PXRD and (E) $\mathrm{N}_{2}$ sorption. 
A

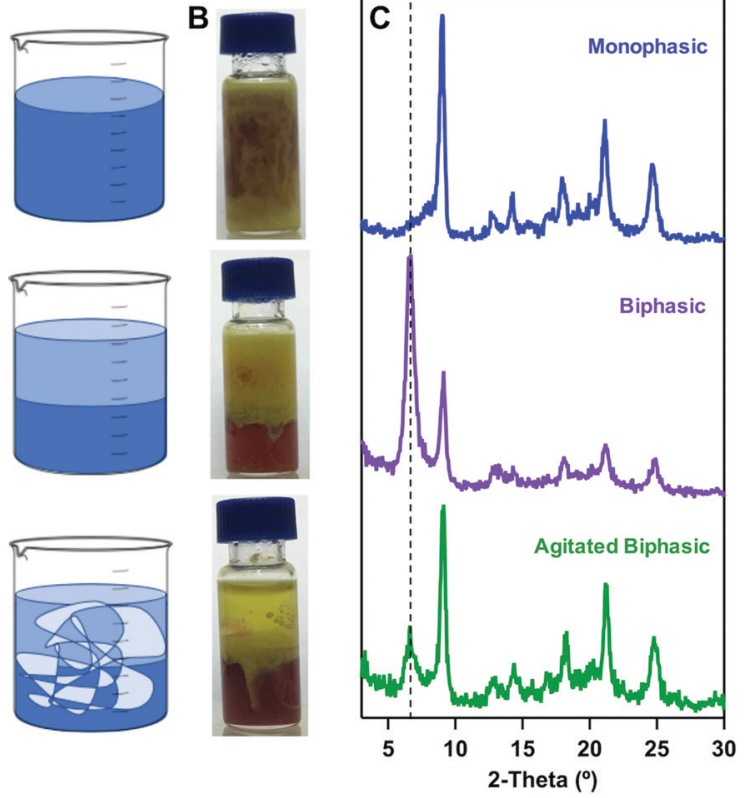

Fig. 3 Modulated COF-300 growth conditions with and without toluene. (A) Graphical representation, (B) growth solution one day at $90{ }^{\circ} \mathrm{C}$, and (C) PXRD of the resulting solid after two days at $90^{\circ} \mathrm{C}$.

easily activated to the ideal form with mild vacuum heating (Fig. S3 and S4, ESI $\dagger$ ). This establishes that isolation and purification of the amorphous solid prior to crystallization rearrangement has a direct impact on the final form of COF-300 generated during synthesis.

With amorphous regrowth identified as a method to preferentially synthesize porous COF-300 over the collapsed form, conditions were designed to better understand the structural directing agents of the amorphous rearrangement. Control experiments confirm that 1,4-dioxane, water, and acetic acid are all required for rearrangement of the amorphous solid to the crystalline form (Fig. S13, ESI $\dagger$ ), indicating that catalyzing imine exchange is necessary. The purification and isolation of the amorphous intermediate expels the residual water and generates a more dense solid, evident by visual inspection of the reaction solutions. We hypothesize that the collapsed COF-300 structure is driven by templating water molecules bound within the swollen amorphous gel, where their strong binding affinity energetically outweighs the framework distortion. To test this, conditions were developed to modulate the hydrophobicity of the growth conditions while still retaining available water for imine exchange. Through the addition of toluene to the initial homogeneous growth solvent (dioxane: toluene $70: 30 \mathrm{v} / \mathrm{v}$ ), a biphasic solvent formed, dividing the TAPM-containing organic layer and the $3 \mathrm{M}$ aqueous acetic acid layer. Upon addition of the PDA to the top organic layer, polymer solids formed rapidly at the solvent interface. After growth for two days at $90{ }^{\circ} \mathrm{C}$, the biphasic conditions provide an increased preference for the porous COF-300 over the collapsed form, based upon PXRD analysis (Fig. 3). As a control, vigorous agitation of the biphasic system prior to PDA addition results in increased initial solid formation in the top organic layer, more similar to monophasic conditions, with a

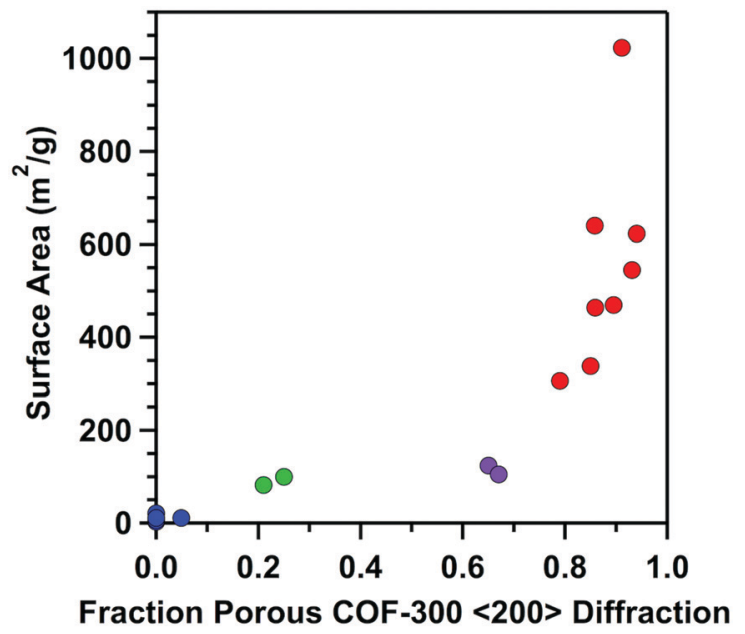

Fig. 4 Surface area of synthesized COF-300 as a function of the relative intensities of $\langle 200\rangle$ diffraction. Synthesis conditions are: monophasic (blue), agitated biphasic (green), biphasic (purple), and monophasic regrowth (red).

corresponding shift towards primarily collapsed COF-300 in the final material. This shift towards collapsed COF-300 is also observed when the biphasic mixture is constantly stirred throughout the reaction (Fig. S15, ESI†). While the initial growth solvent has a strong impact on final crystalline form when starting with soluble monomers, this directing effect is eliminated in an amorphous solid regrowth, where all solvent conditions explored result in formation of the porous COF-300 (Fig. S14, ESI $\dagger$ ). Despite the fact that powder aggregation of the different forms is similar based on SEM characterization (Fig. S16-S21, ESI $\dagger$ ), these results illustrate that the structure of the amorphous solid has a strong control over the crystalline form of COF-300 generated during imine rearrangement.

A comparative analysis of COF- 300 products establishes the relationship between crystal form, surface area, and synthesis conditions (Fig. 4). The ratio of porous to collapsed COF-300, based on the $\langle 200\rangle$ diffraction peak, has a clear impact on the product's surface area, with higher fractions of the porous form resulting in high surface area materials. Growth conditions direct transformation of the intermediate amorphous species into either porous or collapsed COF-300. Biphasic conditions yield an increased fraction of porous COF-300 compared to monophasic conditions, with a corresponding 6-fold increase in surface area, while the agitated biphasic mixture provides intermediate levels. Isolation and regrowth of the amorphous solid generally provides the highest fraction of porous COF-300. As the collapsed COF-300 has structural distortions energetically counterbalanced by the internally bound water, this suggests that the ideal growth conditions require minimizing water binding within the material during crystallization. Since the COF can form over a wide range of porous/collapsed ratios and corresponding surface areas, it is extremely important to control synthesis via the growth conditions.

To establish generality of this approach, similar conditions were applied to the synthesis of related imine-linked 3D COF-320. ${ }^{28}$ The reported structure of COF-320 is nine-fold interpenetrated, with a major powder diffraction $\langle 200\rangle$ peak expected at $5.6^{\circ}$ 

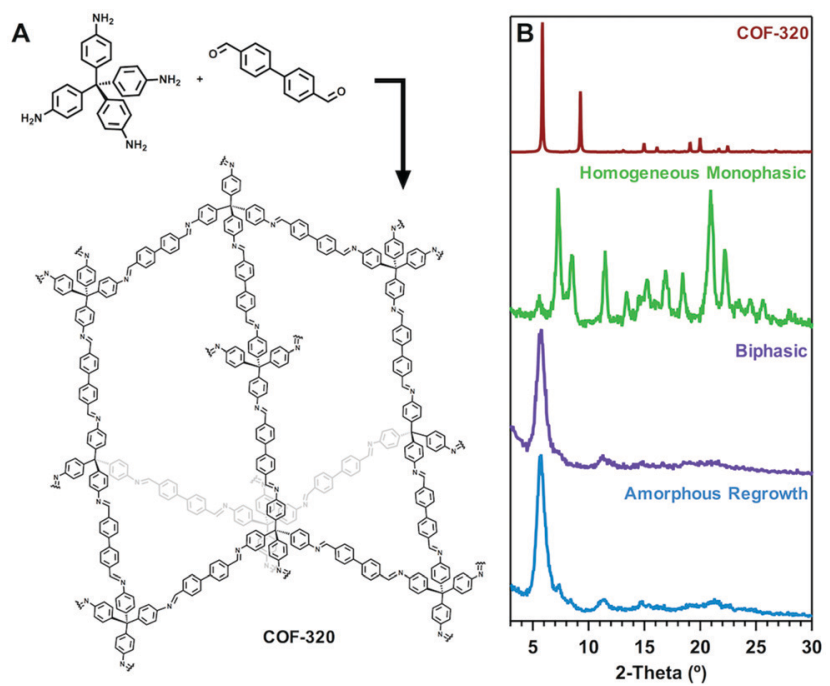

Fig. 5 (A) Scheme of COF-320. (B) Expected PXRD pattern generated from the reported crystal structure (ref. 28), and experimental results under different growth conditions.

$2 \theta$ based on that structure. Synthesis of COF-320 from fully homogenous, monophasic starting conditions yields a crystalline material with a notably different pattern, consistent with a collapsed form (Fig. 5). In contrast, biphasic growth conditions yield the expected powder pattern. Consistent with COF-300, COF-320 forms an initial amorphous solid, which when isolated and re-exposed to growth conditions, generates the ideal COF-320 crystal form. Overall, these results indicate both structural distortion and amorphous intermediates to be general properties of 3D iminelinked COFs, as well as establish growth solvent modulation as a powerful approach to selectively generate different COF structures.

Mechanistic understanding is essential to transition the field of 3D covalent organic frameworks from initial material synthesis to broader applications. This study establishes how to predict and control for the synthesis of both porous and collapsed imine-linked COFs through an amorphous intermediate. We establish a preference for the porous form synthesized through either a two-step isolation/purification process or a one-step biphasic growth environment. We anticipate this approach will be beneficial in the development of applications for known 3D COFs as well as in the discovery of novel systems.

The authors would like to thank Prof. Christopher Daniel for help in SEM data collection. This research was supported by Bucknell University.

\section{Conflicts of interest}

The authors have no conflicts to declare.

\section{Notes and references}

1 M. S. Lohse and T. Bein, Adv. Funct. Mater., 2018, 28, 1705553.

2 F. Beuerle and B. Gole, Angew. Chem., Int. Ed., 2017, 57, $4850-4878$.

3 R. P. Bisbey and W. R. Dichtel, ACS Cent. Sci., 2017, 3, 533-543.

4 N. Huang, P. Wang and D. Jiang, Nat. Rev. Mater., 2016, 1, 16068.

5 P. J. Waller, F. Gándara and O. M. Yaghi, Acc. Chem. Res., 2015, 48, 3053-3063.

6 X. Feng, X. Ding and D. Jiang, Chem. Soc. Rev., 2012, 41, 6010-6022.

7 S.-Y. Ding and W. Wang, Chem. Soc. Rev., 2012, 42, 548-568.

8 Y. Song, Q. Sun, B. Aguila and S. Ma, Adv. Sci., 2018, 1801410.

9 S. Zhang, Q. Yang, C. Wang, X. Luo, J. Kim, Z. Wang and Y. Yamauchi, Adv. Sci., 2018, 1801116.

10 H. Fan, J. Gu, H. Meng, A. Knebel and J. Caro, Angew. Chem., Int. Ed., 2018, 57, 4083-4087.

11 X. Wang, L. Chen, S. Y. Chong, M. A. Little, Y. Wu, W.-H. Zhu, R. Clowes, Y. Yan, M. A. Zwijnenburg, R. S. Sprick and A. I. Cooper, Nat. Chem., 2018, 10, 1180-1189.

12 X. Wang, X. Han, J. Zhang, X. Wu, Y. Liu and Y. Cui, J. Am. Chem. Soc., 2016, 138, 12332-12335.

13 H. Xu, J. Gao and D. Jiang, Nat. Chem., 2015, 7, 905-912.

14 J. Wang, L. Si, Q. Wei, X. Hong, S. Cai and Y. Cai, ACS Appl. Nano Mater., 2018, 1, 132-138.

15 D. A. Vazquez-Molina, G. S. Mohammad-Pour, C. Lee, M. W. Logan, X. Duan, J. K. Harper and F. J. Uribe-Romo, J. Am. Chem. Soc., 2016, 138, 9767-9770.

16 C. R. Mulzer, L. Shen, R. P. Bisbey, J. R. McKone, N. Zhang, H. D. Abruña and W. R. Dichtel, ACS Cent. Sci., 2016, 2, 667-673.

17 S. Duhović and M. Dincă, Chem. Mater., 2015, 27, 5487-5490.

18 F. Xu, S. Jin, H. Zhong, D. Wu, X. Yang, X. Chen, H. Wei, R. Fu and D. Jiang, Sci. Rep., 2015, 5, 8225.

19 Z. Li, X. Feng, Y. Zou, Y. Zhang, H. Xia, X. Liu and Y. Mu, Chem. Commun., 2014, 50, 13825-13828.

20 M. G. Rabbani, A. K. Sekizkardes, Z. Kahveci, T. E. Reich, R. Ding and H. M. El-Kaderi, Chem. - Eur. J., 2013, 19, 3324-3328.

21 J. L. Segura, M. J. Mancheño and F. Zamora, Chem. Soc. Rev., 2016, 45, 5635-5671.

22 T. Ma, E. A. Kapustin, S. X. Yin, L. Liang, Z. Zhou, J. Niu, L.-H. Li, Y. Wang, J. Su, J. Li, X. Wang, W. D. Wang, W. Wang, J. Sun and O. M. Yaghi, Science, 2018, 361, 48-52.

23 D. Beaudoin, T. Maris and J. D. Wuest, Nat. Chem., 2013, 5, 830-834.

24 B. J. Smith and W. R. Dichtel, J. Am. Chem. Soc., 2014, 136, $8783-8789$.

25 B. J. Smith, N. Hwang, A. D. Chavez, J. L. Novotney and W. R. Dichtel, Chem. Commun., 2015, 51, 7532-7535.

26 B. J. Smith, A. C. Overholts, N. Hwang and W. R. Dichtel, Chem. Commun., 2016, 52, 3690-3693.

27 F. J. Uribe-Romo, J. R. Hunt, H. Furukawa, C. Klöck, M. O'Keeffe and O. M. Yaghi, J. Am. Chem. Soc., 2009, 131, 4570-4571.

28 Y.-B. Zhang, J. Su, H. Furukawa, Y. Yun, F. Gándara, A. Duong, X. Zou and O. M. Yaghi, J. Am. Chem. Soc., 2013, 135, 16336-16339. 